\title{
p53 and disease: when the guardian angel fails
}

\author{
JA Royds ${ }^{\star, 1}$ and B lacopetta ${ }^{2}$ \\ ${ }^{1}$ Department of Pathology, University of Otago, Dunedin, New Zealand \\ ${ }^{2}$ School of Surgery and Pathology, University of Western Australia, Western \\ Australia, Australia \\ * Corresponding author: JA Royds, Department of Pathology, Dunedin School of \\ Medicine, PO box 913, Dunedin 9001, New Zealand. Tel: + 643 4797471; \\ Fax: + 643479 7136; E-mail: janice.royds@stonebow.otago.ac.nz
}

Received 15.12.05; revised 24.2.06; accepted 24.2.06; published online 24.3.06 Edited by $A$ Braithwaite

\begin{abstract}
The p53 tumor suppressor gene (TP53) is mutated more often in human cancers than any other gene yet reported. Of importance, it is mutated frequently in the common human malignancies of the breast and colorectum and also, but less frequently, in other significant human cancers such as glioblastomas. There is also one inherited cancer predisposing syndrome called Li-Fraumeni that is caused by TP53 mutations. In this review, we discuss the significance of p53 mutations in some of the above tumors with a view to outlining how p53 contributes to malignant progression. We also discuss the usefulness of TP53 status as a prognostic marker and its role as a predictor of response to therapy. Finally, we outline some evidence that abnormalities in p53 function contribute to the etiology of other non-neoplastic diseases.

Cell Death and Differentiation (2006) 13, 1017-1026. doi:10.1038/sj.cdd.4401913; published online 24 March 2006
\end{abstract}

Keywords: p53; prognosis; cancer; Li-Fraumeni syndrome

Abbreviations: GBM, glioblastoma multiforme; IR, ionizing radiation; LFS, Li-Fraumeni syndrome

\section{Introduction}

Our understanding of p53 function gleaned from meticulous in vitro and animal studies continues to increase at a rapid rate, as outlined in the preceding chapters and illustrated by the many talks presented at the most recent International p53 Workshop. A question widely asked by many clinicians who diagnose and treat cancer and other diseases is whether this knowledge can be used to improve the management of their patients. Restoration of normal p53 functions in cancer cells is a promising area of research and is covered in a separate chapter on p53-targeted therapy. ${ }^{1}$ In this chapter, we review other developments in p53 research as they relate to human disease. These include germline p53 mutations and the $\mathrm{Li}$ Fraumeni syndrome (LFS), somatic p53 mutations in cancer and their prognostic and predictive significance, as well as recent evidence suggesting a role for p53 in non-neoplastic diseases.

This is not intended as an exhaustive appraisal of the impact p53 can make in the clinic but as a selection of examples of how failure of regulated p53 function not only leads to disease but can also influence its management.

\section{p53 Germline Mutations and Li-Fraumeni Disease}

p53, famously dubbed 'The Guardian of the Genome', is arguably the most significant gene for cancer suppression. ${ }^{2}$ Somatic loss of function of $p 53$ underpins tumor progression in most epithelial cancers and many others besides. Indeed haploinsufficiency and even certain polymorphisms in TP53or its controlling factor Mdm2 are enough to increase cancer incidence. ${ }^{3}$ What phenotype should we expect therefore in cases of germline loss of this gene? The LFS (MIM 151623) is a clinically and genetically heterogenous autosomal dominant disorder, caused mainly by mutations in the TP53 gene, which gives rise to early-onset cancers. ${ }^{4,5}$ Approximately $80 \%$ of LFS families that fulfill the classical clinical criteria (see Table 1) have mutations in TP53, with loss of p53 pathway function probably accounting for the rest (reviewed by ${ }^{6}$ ). Surprisingly, the tumor spectrum seen in this familial cancer syndrome is quite different from that associated with somatic TP53 mutations. Thus, instead of the epithelial cancers where somatic mutations are prevalent, LFS patients present with rarer tumors such as sarcomas, brain and pre-menopausal breast tumors, adrenocortical carcinomas and leukemias (hence the alternative name of 'sarcoma, brain, leukemia, adrenal' or SBLA syndrome). A significantly higher risk of cancer is associated with female carriers, a difference that may $^{7}$ or may not be due to an excess of breast cancer. ${ }^{8}$ The frequency of de novo mutations is not known, however it may be quite significant as one study found $4 / 17$ cases were owing to new mutations. ${ }^{9}$

In contrast to other inherited cancer syndromes with the characteristic single presenting neoplasm, LFS patients present with a range of tumors. This has led to various clinical definitions of LFS and to the identity of associated syndromes, LF-like (LFL) and incomplete LFS (LFI) with 40 and 6\% germline mutant (mt) TP53, respectively (Table 1).,10,11 CHEK2 (22q12.2) and a locus on 1q23 have been identified as other predisposing LFS loci. ${ }^{12,13}$ CHEK2 codes for checkpoint kinase2, a serine/threonine protein kinase. CHEK2 is a homologue of Rad53 in yeast, required for DNA damage and replication checkpoints. The protein encoded by CHEK2 phosphorylates $\mathrm{p} 53$, thus signaling in response to DNA double strand (ds) breaks, halting mitosis and allowing DNA repair. ${ }^{14}$ Thus, the DNA damage repair pathway is a major barrier to tumor formation, and Chk2 and p53 are key players in signaling. ${ }^{15}$ Pre-neoplastic conditions are associated with DNA replication stress and an activated DNA damage 
response, including phosphorylation of Chk2. Thus, during tumorigenesis, DNA damage signaling, and especially the p53 pathway including Chk2, is under selective pressure for mutation. $^{16}$

Most TP53 germline mutations are the common missense mutations in the DNA binding domain of p53 in codons such as $175,248,249,273$ and 281 . However, the frequency distribution of LFS mutations does differ from the somatic pattern (database of LFS mutations www.iarc.fr/p53). ${ }^{17}$ Amino acids most frequently altered as a result of the germline mutations are $248>273>175>245$, whereas for sporadic mutations the order is $175>248>273>245$. Other germline hotspots are 235, 133 and 181. Moreover, splicing mutations are more frequent in germline than in sporadic mutations, with as many as $15 \%$ reported in one study. ${ }^{18}$ Interestingly, germline TP53 gene deletions are extremely rare, making p53 null mice an imperfect model for LFS. ${ }^{19}$

Recently, the gain-of-function R172H (G to $A$ at nucleotide $515)$ equivalent to 175 in humans has been used to create a mouse model of LFS. ${ }^{20}$ Although $p 53^{+/ 515 A}$ and $p 53^{+/-}$were similar in tumor spectrum, there were significant differences. The gain-of-function phenotype of $\mathrm{p} 53 \mathrm{R} 172 \mathrm{H}$ was associated with poor differentiation and a metastatsis. The nature of the mutation, the interplay between the p53 family members and the genetic background of the host are all important factors in determining the final phenotype. This has clearly been demonstrated in mouse models of LFS, with the 172 mutation giving rise to osteosarcomas and the 270 mutation to carcinomas. ${ }^{20,21}$ Thus, identifying the type of TP53 mutation may be important in the management of LFS families.

Clinical management of LFS is difficult owing to the range of sites, types of cancer and ages of presentation. There appear to be no universally agreed rules for managing LFS but there are some published guidelines. ${ }^{22}$ Clinical genetic counseling for families with germline TP53 mutations is obviously important. Patients and at-risk family members should also be monitored to identify tumors early. Female subjects can be offered prophylactic mastectomy or regular mammograms. Life style counseling on avoiding ionizing radiation (IR) and other DNA-damaging agents should also be given. This increased susceptibility to IR also needs to be borne in mind when treating tumors from young LFS patients, as these individuals are at high risk of second malignancies. ${ }^{23}$ Prenatal or pre-implantation genetic testing can be offered to fertile couples affected by LFS. Moreover, as mentioned above, determining the exact nature of the TP53 mutation may be of prognostic significance and therefore important for the clinical management of these patients.

LFS although perhaps surprisingly rare (approximately 400 families reported worldwide) is a highly penetrant disorder with the lifetime risk of developing cancer being over $90 \%$. However, owing to the difficulty in finding an absolute definition for 'LFS' and the resulting stringent defining clinical features for its diagnosis (Table 1), it is possible that LFS is under reported. The high frequency of new mutations will mean that many patients with germline mutations will go undiagnosed, for a diagnosis of LFS requires a detailed family history. Moreover, different mutations and different host genetic backgrounds impact on the spectrum of tumors arising. This may result in germline TP53 mutations being
Table 1 Characteristics of the LFS

Two forms of LFS:

- Classic

- Li-Fraumeni-like

Classic

LFS is defined by the following criteria ${ }^{4}$

- A proband with a sarcoma diagnosed before 45 years of age plus

- A first-degree relative with any cancer before 45 years of age plus

- One additional first- or second-degree relative with any cancer before the age of 45 years or a sarcoma at any age

Li-Fraumeni-like (LFL)

Two definitions are listed below:

Birch's definition of $L F L^{11}$

- A proband with any childhood cancer or sarcoma, brain tumor or adrenal cortical tumor diagnosed before 45 years of age plus

- A first- or second-degree relative with a typical LFS cancer (sarcoma, breast cancer, brain tumor, adrenal cortical tumor or leukemia) at any age plus

- A first- or second-degree relative with any cancer under the age of 60 years

Eeles' definition of $L F L^{10}$

- Two first- or second-degree relatives with LFS-related malignancies at any age.

The table shows different diagnostic criteria for LFS. Adapted from http:// www.genetests.org

involved in the genesis of some tumors without a diagnosis of LFS being made. For example, germline mutations in TP53 have been recognized in patients with glioma, irrespective of family history. ${ }^{5}$

\section{p53 mutations, ALT and gliomas}

Germline TP53 mutations are associated with glioma and in particular the high-grade glioblastoma multiforme (GBM). ${ }^{5}$ These brain tumors are associated particularly with TP53 mutations in the DNA binding loop that contacts the minor groove $(P=0.01)$ and with null mutations. The latter are associated with earlier onset brain tumors $(P=0.004) .{ }^{17}$ GBMs are the most prevalent primary brain neoplasm in adults and their incidence is inexplicably on the increase. ${ }^{24}$ They have a disproportionate socio-economic ranking compared to their incidence, being the fourth greatest impacting cancer. Treatments are not curative and mortality rates are high, with very little improvement in over 25 years. However, length of survival varies greatly, with the median being 9 months and a small but significant percentage of patients living for 2-3 years post diagnosis. TP53 mutations are associated with GBM and lower-grade gliomas and in particular with their telomere maintenance function ${ }^{25}$ (JA Royds, unpublished data). Telomeres are eroded with every cell division unless repaired by a telomere maintenance mechanism (TMM) such as telomerase or the alternative lengthening of telomeres (ALT) pathway that involves homologous recombination between telomeres. Consistent with this, it has been reported that ALT is repressed by sequestration of the MRE11/RAD50/NBS1 recombination complex by Sp100. ${ }^{26,27}$ ALT is characterized by the presence of long telomeres and ALT-associated PML bodies (APBs; PML bodies containing telomeric DNA and binding proteins). ${ }^{28}$ 
a

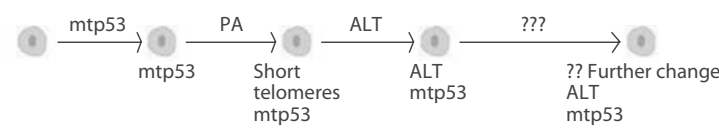

Normal LG or silent $\quad$ AA GBM

Age in years

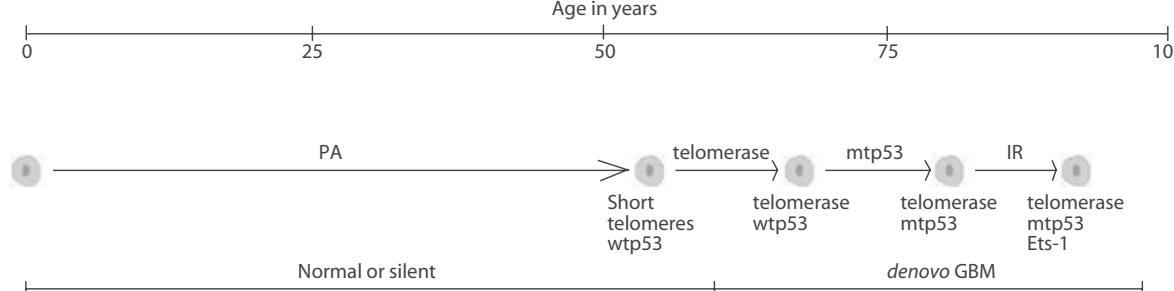

Prognosis

Figure 1 Model for the proposed effects of p53 and TMM on different molecular pathways in gliomagenesis. (a) ALT gliomas. p53 lesions are considered a late occurrence in the tumorigenesis of most carcinomas; however, we postulate that for some gliomas the tumor progenitor cells acquire an early mutation in TP53. This leads to a proliferative advantage (PA) in these cells, resulting in shortened telomeres and acquisition of a TMM. The mutant p53 facilitates selection of the ALT mechanism. The fact that the long and heterogeneous telomeres characteristic of ALT are tolerated suggests that a defect in p53 damage signaling must be in place before ALT can succeed as a telomere maintenance mechanism. We therefore hypothesize that in ALT cells mt TP53 occurs early, with a possible gain-of-function effect. As we find ALT in AA, we suggest that further, unknown changes occur to give a GBM. An early p53 mutation appears to lead to GBM at an earlier median age but with a better prognosis than wt p53 tumors. (b) Progenitor cells, of the same or different lineage from (A), acquire a PA that does not select for mutation in p53. This PA along with other mutations causes a de novo GBM. Telomeres shorten and telomerase is switched on in many cases. Analogous to the common situation for carcinomas, an mt p53 may be selected at late times in a few cases and where it appears to confer a poor prognosis. This may be due to upregulation of Ets-1 expression in these de novo GBM with mtp53. $L G A=$ low-grade astrocytoma, $A A=$ anaplastic astrocytoma, $G B M=$ glioblastoma multiforme, $\mathrm{IR}=$ ionizing radiation

Interestingly, TP53 mutations are also associated with the ALT phenotype $(P=0.001)$ but not with telomerase $(P=0.64)$. Thus, loss of p53 early in tumorigenesis may facilitate selection of a recombinational TMM such as ALT. The most frequent TP53 mutations found in ALT-positive GBMs are at codons 248, 273, 282 and 'nonsense', a pattern that is not dissimilar to LFS. The frequency for non-ALT GBMs is $273 \gg 248>282>220$, with no 'nonsense' mutations observed (JA Royds, unpublished data). Thus, germline mutation or early acquisition of a somatic mutation in TP53 may predispose to the ALT phenotype. It is also interesting to note that fibroblasts from LFS patients often acquire ALT when immortalized. ${ }^{29}$

These findings have important clinical significance, as ALT identifies a sub-type of GBM that has a more favorable prognosis and occurs in younger patients (Figure 1). ${ }^{30}$ In a study of 77 GBM patients, it was found that ALT more than doubled the median survival for patients with GBM (542 versus 247 days). ${ }^{30}$ In a later study, we showed that TP53 mutations are prognostic only in younger patients (median survival 490 versus 316 days, $P=0.01$ ). Interestingly, the longest survival is seen in patients whose GBMs have both ALT and TP53 mutation (651 versus 228 days). In contrast, patients with telomerase and mt TP53 had the shortest survival (127 days) (JA Royds, unpublished data). Thus, evaluation of TMM, along with TP53 mutations, should therefore become an essential component of patient management and the design of clinical trials for GBM therapies. These results suggest that germline TP53 mutations may predispose to the development of a subgroup of high-grade gliomas that occur earlier in life but which have a less aggressive phenotype than the rest. Providing accurate prognosis is vital for the management of glioma patients, and a new technique to identify ALT in archival material has been developed by identifying ALT-associated PML bodies. ${ }^{28}$ These findings may have implications for other LFS tumors. ALT has been demonstrated in sarcomas, another LFS tumor, again at an earlier age, but in the case of sarcomas, ALT does not appear to be related to survival. ${ }^{28}$

Although most ALT gliomas have mt p53, forced expression of wild-type (wt) p53 in ALT cells resulted in rapid inhibition of proliferation and induction of APBs that express p21 Waf1/ cip1. ${ }^{31}$ Thus, at late stages in tumorigenesis, wt p53 does not inhibit ALT per se but does cause cell cycle arrest, probably owing to the presence of DNA damage signals. Thus, wt p53 function and ALT may be largely incompatible for tumor formation. The introduction of wt p53 into ALT tumors may be worth considering as a therapeutic strategy.

The reason why $\mathrm{mt}$ p53 in telomerase-positive GBM signifies a slightly worse prognosis is unknown (median survival wt $\mathrm{p} 53=190$ days and $\mathrm{mt} p 53=123$ days) (JA Royds, unpublished results). This probably relates to their molecular phenotype influencing invasive potential, which makes GBMs such an aggressive tumor. Ets-1 is frequently overexpressed in GBM and this is associated with glioma invasion. $^{32}$ It has been demonstrated that a functional interaction between $\mathrm{p} 53$ and Ets-1 can determine the invasive potential of some glial tumors. Gamma irradiation treatment 
activates wt p53 resulting in downregulation of Ets-1; however, the same treatment can result in Ets-1 up regulation in cells harboring certain mtp53 $(175 \mathrm{H}){ }^{33}$ TP53 mutations found in GBM may have a gain of function, switching on genes involved in the glioblastoma pathway. Thus late acquisition of a TP53 mutation may induce aggressive properties in gliomas, particularly those with telomerase, by upregulation of Ets-1 and other genes leading to an increased propensity for invasion. It is interesting to note that Ets-1 is activated not only by mitogens, but also by hypoxia via hypoxia-inducible factor-1 (hif1) ${ }^{34}$ (summarized in Figure 1).

\section{p53 and Hypoxia}

Hypoxic tumors are more aggressive and harder to treat, and understanding the reasons for this would aid in their management. ${ }^{35}$ Reduced oxygen tension is a feature of rapid cellular growth, featuring in development and in proliferative diseases such as cancer and atherosclerosis. Angiogenesis modulators have been implicated in atherosclerotic disease, facilitating plaque expansions in an analagous fashion to tumor expansion. Neo-angiogenesis, which accompanies these diseases, produces a poorly formed and therefore an ineffective vasculature that often produces cycles of transient ischemia and hypoxia followed by reperfusion and reoxygenation. Reoxygenation after periods of hypoxia leads to DNA damage, which in turn can result in mutation and tumor progression.

Hypoxia is a significant physiological stress affecting malignant progression and leading to p53 accumulation. Graeber $^{36}$ showed that hypoxia-induced apoptosis is p53 mediated. Thus wt p53 tumors underwent extensive apoptosis after hypoxia but p53 null tumors did not. However, there is a lack of evidence for p53 acting in its traditional transcriptional role when activated by hypoxia and it is more likely that changes in transrepression are significant. Thus, hypoxiainduced p53 is unusual in that it fails to transactivate Mdm2 and levels of the latter are low in hypoxic tissues, thus leading to a stabilization of p53. Hypoxia-induced apoptosis involves cytochrome $c$ release requiring Apaf1 and caspase 9 but not Bax. ${ }^{37}$ How then does hypoxia-induced p53 activate apoptosis? It is possible that a proportion of the hypoxia-stabilized p53 acts directly at the mitochondrion to induce apoptosis. ${ }^{38,39}$ However, it is p53's transrepressor function that is the main operator in the induction of apoptosis in low oxygen tension. The reason why hypoxia-induced p53 fails its normal transactivation function is not certain, but it has been hypothesized that Hif1 binds all available coactivators such as $\mathrm{p300/CBP}{ }^{37}$

The transrepressor function of p53 is less well understood but appears to require the polyproline region of p53 (amino acids 64-91). During hypoxia-directed apoptosis, the p53 proline region binds the nuclear corepressor protein $\operatorname{Sin} 3 \mathrm{a}^{40}$ Mutational analysis has highlighted residue 71 as a requirement for binding. ${ }^{40}$ Deletion of the proline-rich region results in loss of apoptosis under hypoxic conditions. Under hypoxic conditions in which p53 is activated, it complexes with Sin3 and HDAC at promoters and blocks transactivation. ${ }^{37}$ The interaction of p53 and $\operatorname{Sin} 3$ at the $\operatorname{Sin} 3$ binding domain (amino acids 62-73) has a stabilizing effect on p53 owing to inhibition of proteosome-mediated destruction. Moreover, it was demonstrated that loss of p53 transrepressor function owing to mutation results in the loss of apoptosis in hypoxic conditions. In particular, mutation of the DNA binding domain residues abolishes repression-mediated apoptosis.

What are the target genes of this hypoxia-induced transcriptional repression? One candidate is survivin, a member of the inhibitor of apoptosis (IAP) family. Survivin is repressed by $\mathrm{p53}$, thus relieving inhibition of effector caspases such as caspase 9. ${ }^{41,42}$ Other anti-apoptotic candidates for hypoxia-induced p53 repression include $\mathrm{Bcl} 2$, Map4 and IGF1 receptor. ${ }^{37}$ It is likely that p53-repressed targets are several and varied according to the prevailing conditions and tissue; thus, more candidate genes need to be identified.

Angiogenesis in response to hypoxia results in inefficient new vessels that are prone to transient collapse resulting in cycles of hypoxia and reoxygenation. This has implications for treatment as inefficient vasculature affects drug delivery and also results in a lack of oxygen in a tumor that reduces levels of oxygen radicals, the active principle behind radiotherapy. Moreover, tumor cells with a mt p53 resist hypoxia-induced apoptosis and are therefore positively selected by this stress. Thus, the combination of these factors offers a plausible explanation for the poor prognostic significance of tumor hypoxia.

To summarize, in contrast to DNA damage, p53 induced by severe hypoxia acts as a transrepressor in the induction of apoptosis. In hypoxia, p53 associates with corepressor molecules such as Sin3a and HDAC.

\section{TP53 Somatic Mutations in Human Cancer - Lessons from p53 Mutation Databases}

Somatic mutations in p53 are found in approximately $50 \%$ of all human cancers. The frequency varies according to tumor type and ranges from about $10 \%$ in leukemia, $20 \%$ in breast cancer, $30 \%$ in gastric cancers and up to $60 \%$ in ovarian and colorectal cancers. Wide variations have been reported between different studies of the same tumor type, probably reflecting methodological, geographic and case mix differences. One of the major issues has been the use of immunohistochemical (IHC) technique as a surrogate marker for p53 abnormality. Concordance between the overexpression of p53 protein detected by $\mathrm{IHC}$ and the presence of gene mutation detected by molecular methods is approximately $70 \% .{ }^{43}$ For reasons of brevity, the current review will focus only on studies of p53 gene mutation, although it should be remembered that p53 overexpression may also have clinical value in some situations.

Investigations into the role of p53 mutation in human cancer have benefited greatly from the compilation of two large databases. ${ }^{44,45}$ These were achieved through the efforts of several diligent individuals and by the contribution of data from many researchers worldwide. Fascinating insights have emerged including links between exposure to various types of carcinogen and the spectrum of p53 mutations. The databases reveal that over $80 \%$ of p53 mutations are missense 
mutations that lead to the synthesis of full-length protein. Approximately 1400 different p53 mutations have been reported, with the large majority localized to the DNA binding domain. Some mutations are observed very frequently (hotspots) and these clearly have strong biological relevance. However, almost $30 \%$ of p53 mutations occur rarely and this has raised questions about their significance. ${ }^{46}$ The functional activity of more than 2000 p53 mutations created by sitedirected mutagenesis has been investigated using an in vitro yeast system for their ability to transactivate downstream genes. While the most frequently occurring TP53 mutations in the databases display a clear loss of transactivation activity for the promoters MDM2, p53R2, BAX, NOXA, GADD45, AIP1, 14-3-3 $\sigma$, WAF1, more than $50 \%$ of the rare mutations have similar activity levels to wt $\mathrm{p} 53 .{ }^{45} \mathrm{~A}$ recent follow-up study by the Ishioka laboratory found no correlation between the transcriptional activity of 179 different p53 mutations and their ability to induce apoptosis. ${ }^{47}$ Interestingly, 17 'super' mutations involving amino acids 121 and 290-292 were identified as having superior ability to induce apoptosis compared to wt p53. The labor-intensive nature of this type of study has so far prevented the functional evaluation of all p53 mutations for their ability to induce apoptosis in mammalian cells. It should be borne in mind however that the apoptotic ability of different p53 mutants is also likely to be influenced by cell type, thus adding another level of complexity.

The heterogeneity in transactivation and apoptotic activity of different p53 mutations has major implications for clinical studies, as it can no longer be assumed that all p53 mutations are functionally equivalent. Notwithstanding this sobering revelation, the following sections will attempt to summarize the current status regarding the prognostic and predictive significance of $\mathrm{p} 53$ mutation in human cancer.

\section{Prognostic Significance of p53 Mutation in Cancer - Does it Affect Tumor Aggressiveness?}

Considerable optimism initially surrounded the possibility that p53 mutation status could provide prognostic information for the outcome of cancer patients that was independent of conventional TNM histopathological parameters (Table 2). The presence of TP53 mutation would be expected to correlate with a more aggressive phenotype, and indeed a higher frequency of mutation in advanced stage tumors was found in a recent collaborative study involving 3583 colorectal cancers. ${ }^{48}$ A study of breast cancers found that $\mathrm{p} 53$ mutation frequency was not related to nodal involvement or tumor size, ${ }^{49}$ although another study found a marginally increased frequency in recurrent tumors. ${ }^{50}$

The many studies to investigate prognostic significance of p53 mutation in specific tumor types have often yielded inconsistent results. Reasons for this are likely to include the methodology used to detect mutations, small sample size, short length of patient follow-up, use of adjuvant therapies and variable case mix. Meta-analyses combine the results from individual studies in order to produce more reliable estimates of the prognostic significance of p53 mutation. For breast cancer, a meta-analysis of 11 studies involving 2319 unselected cases found a relative risk (RR) for worse survival associated with p53 mutation of $2.0(95 \% \mathrm{Cl}: 1.7-2.5){ }^{51}$ The RR for node-negative breast cancers (1.7, 95\% Cl: 1.2-2.3) was lower than that for node-positive cases (2.6, 95\% Cl: $1.7-$ 3.9) but remained significant. TP53 mutation also showed independent prognostic value for worse survival in nonadjuvant ( $\mathrm{RR}=2.2,95 \% \mathrm{Cl}: 1.2-4.2)$ and adjuvant treated $(\mathrm{RR}=2.0,95 \% \mathrm{Cl}: 1.3-3.1)$ breast cancer patients. ${ }^{52}$ The former result suggests that p53 mutation could be used for the identification of early stage breast cancer patients with very good prognosis and therefore may not require adjuvant therapy. Miller ${ }^{53}$ has shown that a p53-associated transcriptional fingerprint predicts p53 mutational status and is an accurate method for determining prognosis in patients with breast cancer.

Several studies have reported that different types of p53 mutation affecting for example the DNA or zinc binding domains are associated with different prognosis in breast cancer. ${ }^{54,55,56}$ The in vitro functional studies mentioned earlier $^{57}$ lend support to the contention that different p53 mutations may be associated with different phenotypic properties, including tumor aggressiveness. However, another possibility is that any observed differences in patient

Table 2 Prognostic and predictive value of TP53 status

\begin{tabular}{lrl}
\hline Tumor type & Number & TP53 mutation is associated with: \\
\hline Breast & 2319 & Worse survival \\
Breast & 2319 & Node negative disease \\
Breast & 63 & Therapeutic resistance \\
Breast & 90 & Therapeutic resistance \\
Breast & 35 & Therapeutic resistance \\
Breast & 251 & Poor prognosis and therapeutic response \\
Brain & 140 & Young age \\
Brain & 127 & Good prognosis in telomerase-negative patients \\
Colorectal & 18766 & Increase risk of death for 'good' prognosis group \\
Colorectal & 3583 & Advanced stage disease \\
Ovarian & 68 & Resistance to cisplatin \\
Rectal & 1740 & Lack of benefit from 5 fluorouracil treatment \\
& & 50 \\
\hline
\end{tabular}

A summary of the data from the studies discussed in this review is given. The frequency of TP53 mutations in tumors can be found at the website www.iarc.fr.p53 
outcome between different p53 mutations merely reflect differential response to chemotherapy (see below). Carefully designed studies are required to distinguish between these two possibilities.

Colorectal cancer is the other major tumor type to have received considerable attention in terms of the prognostic significance of p53 mutation. A recent systematic review identified 168 reports on p53 abnormality and outcome ${ }^{58}$, although many of these were based on the use of IHC. For studies that used mutation analysis, the increased risk of death associated with p53 mutation in the overall group was $1.31(95 \% \mathrm{Cl}: 1.19-1.45)$ and even higher for patients classified as having good prognosis $(\mathrm{RR}=1.63,95 \% \mathrm{Cl}$ : 1.40-1.90). The CRC-P53 International Collaborative Study evaluated data from 25 different groups for the prognostic and predictive significance of TP53 mutation in colorectal cancer. ${ }^{48}$ This study found differences in prognostic value according to tumor site, with TP53 mutations in proximal and distal colon tumors showing marginal associations with worse outcome ( $R R=1.19$ and 1.29 , respectively) but not those in rectal cancers $(R R=0.97)$.

In summary, there is strong evidence in breast cancer that p53 mutation is an independent factor for poor prognosis. This result is complicated however by the heterogeneity of p53 mutations for the properties of transactivation and induction of apoptosis. The functional heterogeneity of mutations and the costs involved in screening are major obstacles to the introduction of p53 mutation for the routine management of cancer. p53 mutation is only weakly associated with the outcome of colorectal cancer patients and perhaps only in those with good prognosis or with colon tumors. The prognostic significance of p53 mutation in other common cancer types such as ovarian and gastric has not been evaluated to the same level of evidence as breast and colorectal tumors. Major limitations in the interpretation of data are the variable use of adjuvant therapies and the analysis of tumors from different stages.

\section{Predictive Significance of p53 Mutation in Cancer - Does it Affect Tumor Response to Chemotherapy and Radiotherapy?}

p53 is a central mediator of responses to DNA damage and cellular stress and would therefore be expected to play major roles in determining not only the level of tumor aggressiveness but also of chemosensitivity and radiosensitivity. ${ }^{56}$ The former role relates to the prognostic significance of $p 53$, whereas the latter refers to its predictive value. The cellular mechanisms underpinning both roles have been dealt with extensively elsewhere and will not be detailed here. Instead, this section will review the clinical data concerning p53 mutation as a predictive marker for tumor response to adjuvant therapies.

Firstly, it is important to consider study design when evaluating either the prognostic or predictive significance of p53 mutation. Researchers often make the mistake of investigating prognostic significance in patient cohorts that are heterogeneous with respect to adjuvant treatments. This becomes a major confounding factor when tumors with wt p53 are more (or less) responsive to adjuvant treatments compared to those with $\mathrm{mt}$ p53. For example, p53 mutation may have no prognostic significance in patients treated by surgery alone, but a significant prognostic impact might be observed for patients treated with chemotherapy if the subgroup with wt p53 were to derive a survival benefit from this treatment. The variable use of chemotherapy is likely to account for some of the discordant results seen in the literature for the prognostic value of p53 mutation and hence it is recommended that homogeneous treatment groups be investigated at all times.

Whereas the evaluation of prognostic significance is best achieved using homogeneously treated patients, the assessment of predictive value for p53 mutation involves study of patient groups that are treated with or without adjuvant therapy. Randomized prospective trials based on known TP53 status have not been carried out and instead researchers have evaluated predictive significance by using archival tumor blocks from previous clinical trials and retrospectively screened for TP53 mutation. Clearly, there could be major advantages in screening for p53 mutation prior to randomization in future clinical trials, particularly in light of increased knowledge on the functional activities of different mutations. ${ }^{47,57}$

TP53 mutations are reported to predict resistance to anthracycline, ${ }^{54}$ doxorubicin ${ }^{59}$ and 5-fluorouracil/mitomycin ${ }^{60}$ in breast cancer. Mutations affecting the L2 and L3 loop structures in particular were associated with lack of response to chemotherapy. These results support the analysis of TP53 mutation in prospective trials of new chemotherapeutic agents, with special consideration of the structural and functional consequences of mutation. A large, ongoing phase III clinical trial (EORTC 10994/BIG 00-1) of neoadjuvant chemotherapy given before breast cancer surgery has in fact incorporated TP53 mutation analysis into its study design. For colorectal cancer, TP53 mutation was shown in multivariate analysis to be an independent predictor for lack of survival benefit from 5-fluorouracil. ${ }^{61}$ The CRC-P53 International Collaborative Study found that this was particularly evident for rectal cancer. ${ }^{48}$ These clinical observations support in vitro and animal studies showing that colorectal cancer cell lines with inactivated p53 were strikingly resistant to the effects of 5 -fluorouracil. ${ }^{62}$ A clinical study of ovarian cancers also provides evidence that p53 mutations are associated with resistance to cisplatin-based chemotherapy. ${ }^{63}$

In conclusion, there is ample in vitro and clinical evidence to show that p53 mutation is associated with resistance to chemotherapy in several cancer types. These findings support the introduction of TP53 mutation analysis in future clinical trials, possibly using high-throughput functional assays or microarray technology in order to reduce costs. Data from large clinical studies together with results from in vitro studies ${ }^{47,57}$ should allow the predictive value of different TP53 mutations to be assessed, thus opening the way for individualization of patient treatments according to p53 status. Other p53-related genetic factors such as polymorphisms in p53 and MDM2 may also influence response to treatment and require further study. 


\section{TP53 and Inflammation}

p53 is a suppressor of the inflammatory response in mice. In inflammatory conditions, $\mathrm{p} 53^{-1-}$ mice have an increased recruitment of macrophages and a prolonged activation of neutrophils. ${ }^{64}$ As a converse to this, it has been shown that pro-inflammatory macrophage migration inhibitory factor (MIF) interferes with p53 function, thus providing a link between inflammation, atherosclerosis and cancer. ${ }^{65} \mathrm{MIF}$ is overexpressed in tumors and correlates with tumor aggression and downregulation of p53 apoptosis. ${ }^{66}$ MIF regulates E2F function; thus, upregulation of MIF in chronic inflammatory conditions may inhibit p53 response to DNA damage and predispose to acquisition of gene mutations. These findings also have implications for neoplastic transformation of inflammatory epithelia such as in ulcerative colitis (UC) as described below.

\section{Cancer}

Chronic inflammation predisposes to cancer, possibly mediated via genomic instability; for example, UC is a risk factor for colonic cancer. ${ }^{67}$ Approximately 20000 bp/cell/day are oxidized owing to reactive oxygen species (ROS) and this has the potential for generation of point mutations. p53 has an important function in inhibiting the resultant DNA damage. Interestingly, gain of p53 in the form of trisomy $17 p$ has been reported in branching crypts in UC colonic mucosa.

p53 acts to minimize inflammation and its associated DNA damage in several ways. p53 is a potent inhibitor of the pro-

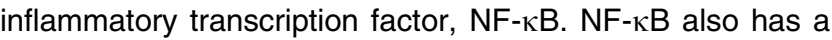
stimulatory role in cancer probably owing to its anti-apoptotic functions. ${ }^{68}$ Drugs that inhibit activation of NF- $\kappa B$ are in use for protecting 'at-risk' patients with inflammatory conditions. p53 also protects against DNA damage induced by chronic exposure to ROS produced by inflammatory cells. It has been shown that p53, even in the absence of stress-induced stabilization, can inhibit oxidative damage to DNA. ${ }^{69}$ p53 mobilizes anti-oxidant defense mechanisms to reduce ROS and associated DNA damage by upregulating glutathione peroxidase, and the sestrins Hi95 and PA26. Inhibition of p53 results in an increase in intracellular ROS and DNA oxidation leading to an increase in mutations. Stress-activated p53 on the other hand increases ROS as part of the apoptotic response. Thus, p53 acts as a protective anti-oxidant until activated by stress whereupon it has a strong oxidative role.

\section{Non-neoplastic diseases}

Although most studies concentrate on TP53 as a tumor suppressor gene, it is inconceivable that p53, a protein that regulates cellular response to stress, is only involved in tumor suppression. Evidence of a role for p53 in other diseases is now beginning to accumulate. Therapeutic manipulation of p53 in these non-neoplastic diseases, which have a stable genome, may yield interesting results, (also discussed by Levine et al., in this issue).

Atherosclerosis underpins most of the world's deadliest non-neoplastic diseases such as coronary heart disease and stroke. Atherosclerosis is a lipid-induced fibroproliferative disease of the arterial intima, complicated by inflammation and macrophage activity. Aberrant growth and migration of human vascular smooth muscle cells (VSMCs) is implicated in the pathogenesis of atherosclerosis. p53 inhibits aberrant intima formation by suppressing migration of VSMCs from the media and by inducing apoptosis in proliferating VSMCs. The significance of this is reflected in the fact that both p53 and p73 are overexpressed in the smooth muscle cells and macrophages of atherosclerotic tissue. ${ }^{70,71}$ Dysfunction of p53 has been linked to the development of unstable and potentially dangerous atheromatous lesions The processes underpinning the evolution of fibrofatty atheromatous lesions from the safer fibrotic type to the lipid-rich unstable plaques are uncertain. In a study by Lavezzi ${ }^{72}$ upregulation and stabilization of p53, Fos and PCNA was observed in the most unstable carotid plaques from patients with stroke or transient ischemic attacks but not in stable carotid lesions from asymptomatic patients. This suggests that p53 plays a role in active plaques, possibly reducing DNA damage in the presence of ROS. p53 also has a role in attempting to stabilize plaques by reducing inflammation and the division and migration of VSMCs and macrophages. Hypoxia and angiogenesis modulation are also involved in plaque expansion and p53 plays a suppressive role here too. Absence of p53 accelerates atherosclerosis in the apoE ${ }^{-1-}$ mouse model by increasing cell proliferation. ${ }^{73}$ In this mouse model for familial hypercholesterolemia, loss of p53 stimulated proliferation in macrophages and was associated with unstable lesions, increased necrosis and with reduced collagen deposition, all bad prognostic features. ${ }^{74}$ Macrophage migration into the fatty core of the plaque plays an important role in the genesis of dangerous lesions.

It is now recognized that inflammatory responses with their increased levels of ROS lead to the dangerously unstable atheromatous plaques that trigger acute and often fatal cerebrovascular and cardiovascular episodes. Downregulation of p53 function (e.g. by MIF) leads to the loss of suppression of $\mathrm{NF}-\kappa \mathrm{B}$, a key signaling molecule in the inflammatory response. (It is interesting to note that $\mathrm{p} 53^{-1-}$ mice are also more susceptible to septic shock.) Thus, activation of $\mathrm{p} 53$ or suppression of the NF- $\mathrm{KB}$ pathway may offer targets for therapeutic intervention in atherosclerosis and other pathological inflammatory conditions. Anti-cancer treatments such as IR may also be applied to atherosclerosis, in particular in the prevention of restenosis after surgical intervention such as angioplasty.

In summary, p53 negatively regulates growth and migration of VSMCs and macrophages, thus minimizing inappropriate accumulation in arterial walls. p53 also has a suppressive effect on the inflammatory process. Thus, loss of p53 function in atheromatous plaques causes increased cellular proliferation, macrophage migration, poor collagen deposition and increased inflammation, all of which cause formation of unstable lesions. In the modified VSMCs of plaques, as in fibroblasts, p53 acts mainly in cell cycle arrest mode (it is interesting to note that $\mathrm{mt}$ p53 is rare in fibrosarcomas, tumors arising from fibroblasts). Thus, activation of p53 and induction of cell cycle arrest in VSMCs may have the effect of increasing collagen synthesis, something that occurs more effectively in non-dividing cells. 


\section{p53 and Degenerative Non-Neoplastic Disorders}

\section{Neurological diseases}

p53 is associated with several neurodegenerative diseases in its capacity to induce cell death in response to stress. Oxidative stress and stimulation by excitatory amino acids (excitotoxicity) activate p53 resulting in neuronal degeneration and death. Oxidative stress is associated with many pathological conditions resulting from neuronal damage including reperfusion injury in stroke or following blood vessel clamping during surgery. Sublethal levels of toxicity result in stabilization of p53 and neuronal dysfunction and degeneration in the absence of total cell removal by apoptosis. Thus, localized activation of caspases by $p 53$ could lead to cellular dysfunction and dendritic loss rather than total neuronal abalation by apoptotic death. p53 is upregulated in damaged neurons containing the neurofibrilary tangles, characteristic of Alzheimer's disease. In the mouse model for Parkinson's disease, concomitant loss of p53 reduces Purkinje cell death and loss of molecular neurons in the cerebellum. The p53 inhibitors pifithrin-a and z-1-117 have been shown to be effective in reducing dopaminergic neuronal loss in this model of Parkinson's disease. ${ }^{75}$

p53 also plays a role in the etiology of ataxias. Many neurodegenerative disorders are associated with a defect in the DNA damage response. Inherited mutations in the ATM gene, which codes for a protein upstream of p53 in signaling DNA damage, cause the autosomal recessive disorder of ataxia-telangectasia. ${ }^{76}$ ATM, a member of the phosphoinositide 3-kinase family, recognizes DNA ds breaks and stabilizes p53. A defective p53 response in this neurological disorder due to a deficiency in this signaling causes a defective cell cycle arrest and apoptotic responses to DNA damage. A patient presenting with ataxia plus oculomotor apraxia (AOA), a common clinical phenotype in many neurological disorders, was found to have a defective stabilization of p53 in response to IR. ${ }^{77}$ The patient's cells were defective in p21 ${ }^{\text {waf1 }}$ induction and in apoptosis and there was also a marked failure to stabilize p73.

Interestingly, several neurological disorders are caused by expansion of trinucleotide repeats. The archetype for these triplet repeat syndromes is Huntington's disease (HD), a progressive neurodegenerative disorder caused by expansion of a polyglutamine tract in the mt huntingtin protein. p53 has now been shown to upregulate huntingtin expression, which may impact on the clinical course of HD. ${ }^{78}$ Thus, stress factors that activate p53 could increase mt huntingtin expression, leading to an earlier onset of the disease. The arginine polymorphism at codon 72 of TP53, which is more effective in apoptosis than the proline variant, also reduces the age of onset of symptoms. ${ }^{79}$ Interaction between p53 and the mt huntingtin protein also decreases p21 Waf1/Cip1 transactivation. A further connection between p53 and HD may involve the ability of p53 to bind to DNA triplet repeats in a structure-selective fashion and to stabilize such tracts to maintain genomic integrity. ${ }^{80}$ Examination of the role of p53 in the stabilization of other potentially recombinogenic repeat structures may prove to be a productive line of investigation.
In summary, p53 induces either repair or cell death in response to cell damage and therefore deficiency of the former or increase of the latter is implicated in the pathology of degenerative disorders. Thus, several diseases or ageinduced alterations in cells could converge on the p53 apoptotic pathway leading to degeneration of non-renewable cell types such as neurons. p53 inhibitors could have therapeutic potential for conditions where p53 can be shown to have a pivotal role. Clearly, further studies are required to determine the mode of action of p53 in HD and other neurological disorders.

\section{Summary and Conclusions}

Thus p53 is implicated in the pathogenesis of the Western world's most common non-infectious diseases, atherosclerosis neurodegeneration and cancer. At present, most of this knowledge is confined to the bench, but as we discover more of the underlying mechanisms involved, p53 will become increasingly important at the bedside.

For cancer patients, inhibition of p53 in normal tissues might reduce side effects from $\mathrm{p} 53$ apoptotic response to genotoxic cancer therapy or mt p53 as a tumor-specific molecule might provide a selective target for treatment. For the aging population, identifying people at risk is important and it may become possible to offer lifestyle advice based on effectiveness of p53 function.

Thus to give David Lane the last word, his prediction that 'Polymorphic variations in p53 response will govern aging and cancer phenotypes' rings true as p53 protects not only from cancer but also atherosclerosis and related diseases.

\section{References}

1. Wiman K (2006) Strategies for therapeutic targeting of the p53 pathway in cancer. Cell Death Differ. in press.

2. Lane DP (1992) Cancer. p53, guardian of the genome. Nature 358: 15-16.

3. Bond GL, Hu W and Levine A (2005) A single nucleotide polymorphism in the MDM2 gene: from a molecular and cellular explanation to clinical effect. Cancer Res. 65: 5481-5484.

4. Li FP and Fraumeni Jr JF (1969) Soft-tissue sarcomas, breast cancer, and other neoplasms. A familial syndrome? Ann. Intern. Med. 71: 747-752.

5. Li YJ, Sanson M, Hoang-Xuan K, Delattre JY, Poisson M, Thomas G and Hamelin R (1995) Incidence of germ-line p53 mutations in patients with gliomas. Int. J. Cancer 64: 383-387.

6. Varley JM (2003) Germline TP53 mutations and Li-Fraumeni syndrome. Hum. Mutat. 21: 313-320.

7. Chompret A (2002) The Li-Fraumeni syndrome. Biochimie 84: 75-82.

8. Hwang SJ, Lozano G, Amos Cl and Strong LC (2003) Germline p53 mutations in a cohort with childhood sarcoma: sex differences in cancer risk. Am. J. Hum. Genet. 72: 975-983.

9. Chompret A, Brugieres L, Ronsin M, Gardes M, Dessarps-Freichey F, Abel A, Hua D, Ligot L, Dondon MG, Bressac-de Paillerets B, Frebourg T, Lemerle J, Bonaiti-Pellie C and Feunteun J (2000) P53 germline mutations in childhood cancers and cancer risk for carrier individuals. Br. J. Cancer 82: 1932-1937.

10. Eeles RA (1995) Germline mutations in the TP53 gene. Cancer Surv. 25: 101124.

11. Birch JM, Hartley AL, Tricker KJ, Prosser J, Condie A, Kelsey AM, Harris M, Jones PH, Binchy A and Crowther D et al. (1994) Prevalence and diversity of constitutional mutations in the p53 gene among 21 Li-Fraumeni families. Cancer Res. 54: 1298-1304. 
12. Bachinski LL, Olufemi SE, Zhou X, Wu CC, Yip L, Shete S, Lozano G, Amos Cl Strong LC and Krahe R (2005) Genetic mapping of a third Li-Fraumen syndrome predisposition locus to human chromosome 1q23. Cancer Res. 65: 427-431.

13. Bell DW, Varley JM, Szydlo TE, Kang DH, Wahrer DC, Shannon KE, Lubratovich M, Verselis SJ, Isselbacher KJ, Fraumeni JF, Birch JM, Li FP, Garber JE and Haber DA (1999) Heterozygous germ line hCHK2 mutations in Li-Fraumeni syndrome. Science 286: 2528-2531.

14. Searle JS and Sanchez Y (2004) Stopped for repairs: a new role for nutrient sensing pathways? Cell Cycle 3: 865-868.

15. Gorgoulis VG, Vassiliou LV, Karakaidos $P$, Zacharatos $P$, Kotsinas A, Liloglou $T$, Venere M, Ditullio Jr RA, Kastrinakis NG, Levy B, Kletsas D, Yoneta A, Herlyn M, Kittas C and Halazonetis TD (2005) Activation of the DNA damage checkpoint and genomic instability in human precancerous lesions. Nature 434: 907-913.

16. Bartkova J, Horejsi Z, Koed K, Kramer A, Tort F, Zieger K, Guldberg P, Sehested M, Nesland JM, Lukas C, Orntoft T, Lukas J and Bartek J (2005) DNA damage response as a candidate anti-cancer barrier in early human tumorigenesis. Nature 434: 864-870.

17. Olivier M, Goldgar DE, Sodha N, Ohgaki $\mathrm{H}$, Kleihues $\mathrm{P}$, Hainaut $\mathrm{P}$ and Eeles RA (2003) Li-Fraumeni and related syndromes: correlation between tumor type, family structure, and TP53 genotype. Cancer Res. 63: 6643-6650.

18. Varley JM, McGown G, Thorncroft M, Kelsey AM and Birch JM (2001) Significance of intron 6 sequence variations in the TP53 gene in Li-Fraumen syndrome. Cancer Genet. Cytogenet. 129: 85-87.

19. Bougeard G, Charbonnier F, Moerman A, Martin C, Ruchoux MM, Drouot N and Frebourg $T$ (2003) Early onset brain tumor and lymphoma in MSH2deficient children. Am. J. Hum. Genet. 72: 213-216.

20. Iwakuma T, Lozano G and Flores ER (2005) Li-Fraumeni syndrome: a p53 family affair. Cell Cycle 4: 865-867.

21. Lang GA, Iwakuma T, Suh YA, Liu G, Rao VA, Parant JM, Valentin-Vega YA Terzian T, Caldwell LC, Strong LC, El-Naggar AK and Lozano G (2004) Gain of function of a p53 hot spot mutation in a mouse model of Li-Fraumeni syndrome. Cell 119: 861-872.

22. Varley JM, Evans DG and Birch JM (1997) Li-Fraumeni syndrome - a molecular and clinical review. Br. J. Cancer 76: 1-14.

23. Limacher JM, Frebourg T, Natarajan-Ame S and Bergerat JP (2001) Two metachronous tumors in the radiotherapy fields of a patient with Li-Fraumeni syndrome. Int. J. Cancer 96: 238-242.

24. Kleihues P and Sobin LH (2000) World Health Organization classification of tumors. Cancer 88: 2887.

25. Batchelor TT, Betensky RA, Esposito JM, Pham LD, Dorfman MV, Piscatelli N, Jhung S, Rhee D and Louis DN (2004) Age-dependent prognostic effects of genetic alterations in glioblastoma. Clin. Cancer Res. 10: 228-233.

26. Jiang WQ, Zhong ZH, Henson JD, Neumann AA, Chang AC and Reddel RR (2005) Suppression of alternative lengthening of telomeres by Sp100-mediated sequestration of the MRE11/RAD50/NBS1 complex. Mol. Cell. Biol. 25: 27082721.

27. Jeyapalan JN, Varley H, Foxon JL, Pollock RE, Jeffreys AJ, Henson JD, Redde RR and Royle NJ (2005) Activation of the ALT pathway for telomere maintenance can affect other sequences in the human genome. Hum. Mol. Genet. 14: 1785-1794.

28. Henson JD, Hannay JA, McCarthy SW, Royds JA, Yeager TR, Robinson RA, Wharton SB, Jellinek DA, Arbuckle SM, Yoo J, Robinson BG, Learoyd DL, Stalley PD, Bonar SF, Yu D, Pollock RE and Reddel RR (2005) A robust assay for alternative lengthening of telomeres in tumors shows the significance of alternative lengthening of telomeres in sarcomas and astrocytomas. Clin. Cancer Res. 11: 217-225.

29. Bryan TM, Englezou A, Dalla-Pozza L, Dunham MA and Reddel RR (1997) Evidence for an alternative mechanism for maintaining telomere length in human tumors and tumor-derived cell lines. Nat. Med. 3: 1271-1274.

30. Hakin-Smith V, Jellinek DA, Levy D, Carroll T, Teo M, Timperley WR, McKay MJ, Reddel RR and Royds JA (2003) Alternative lengthening of telomeres and survival in patients with glioblastoma multiforme. Lancet 361: 836-838.

31. Reddel R (2004) Immortalization via alternative lengthening of telomeres. 12th International p53 Workshop.

32. Yan S, Berquin IM, Troen BR and Sloane BF (2000) Transcription of human cathepsin B is mediated by Sp1 and Ets family factors in glioma. DNA Cell Biol. 19: 79-91.
33. Kim E (2004) Functional interaction between tumor suppressor p53 and protooncogene ETS-1 determines the invasive potential of glial tumors. 12th International p53 Workshop.

34. Ogata N, Ando A, Uyama M and Matsumura M (2001) Expression of cytokines and transcription factors in photocoagulated human retinal pigment epithelial cells. Graefes Arch. Clin. Exp. Ophthalmol. 239: 87-95.

35. Hockel M, Schlenger K, Hockel S and Vaupel P (1999) Hypoxic cervical cancers with low apoptotic index are highly aggressive. Cancer Res. 59: 45254528.

36. Graeber TG, Peterson JF, Tsai M, Monica K, Fornace Jr AJ and Giaccia AJ (1994) Hypoxia induces accumulation of p53 protein, but activation of a G1phase checkpoint by low-oxygen conditions is independent of p53 status. Mol. Cell. Biol. 14: 6264-6277.

37. Hammond EM and Giaccia AJ (2005) The role of p53 in hypoxia-induced apoptosis. Biochem. Biophys. Res. Commun. 331: 718-725.

38. Moll UM and Zaika A (2001) Nuclear and mitochondrial apoptotic pathways of p53. FEBS Lett. 493: 65-69.

39. Sansome C, Zaika A, Marchenko ND and Moll UM (2001) Hypoxia death stimulus induces translocation of p53 protein to mitochondria. Detection by immunofluorescence on whole cells. FEBS Lett. 488: 110-115.

40. Zilfou JT, Hoffman WH, Sank M, George DL and Murphy M (2001) The corepressor mSin3a interacts with the proline-rich domain of p53 and protects p53 from proteasome-mediated degradation. Mol. Cell. Biol. 21: 3974-3985.

41. Hoffman WH, Biade S, Zilfou JT, Chen J and Murphy M (2002) Transcriptional repression of the anti-apoptotic survivin gene by wild type p53. J. Biol. Chem. 277: 3247-3257.

42. Zangemeister-Wittke $U$ and Simon HU (2004) An IAP in action: the multiple roles of survivin in differentiation, immunity and malignancy. Cell Cycle 3 : 1121-1123.

43. Soong R, Robbins PD, Dix BR, Grieu F, Lim B, Knowles S, Williams KE, Turbett GR, House AK and lacopetta BJ (1996) Concordance between p53 protein overexpression and gene mutation in a large series of common human carcinomas. Hum. Pathol. 27: 1050-1055

44. Olivier M, Eeles R, Hollstein M, Khan MA, Harris CC and Hainaut P (2002) The IARC TP53 database: new online mutation analysis and recommendations to users. Hum. Mutat. 19: 607-614.

45. Soussi T, Kato S, Levy PP and Ishioka C (2005) Reassessment of the TP53 mutation database in human disease by data mining with a library of TP53 missense mutations. Hum. Mutat. 25: 6-17.

46. Soussi T and Beroud C (2003) Significance of TP53 mutations in human cancer: a critical analysis of mutations at $\mathrm{CpG}$ dinucleotides. Hum. Mutat. 21: 192-200.

47. Kakudo Y, Shibata H, Otsuka K, Kato S and Ishioka C (2005) Lack of correlation between p53-dependent transcriptional activity and the ability to induce apoptosis among 179 mutant p53s. Cancer Res. 65: 2108-2114.

48. Russo A, Bazan V, lacopetta B, Kerr D, Soussi T and Gebbia N (2005) The TP53 colorectal cancer international collaborative study on the prognostic and predictive significance of p53 mutation: influence of tumor site, type of mutation, and adjuvant treatment. J. Clin. Oncol 23: 7518-7528.

49. Soong R, lacopetta BJ, Harvey JM, Sterrett GF, Dawkins HJ, Hahnel R and Robbins PD (1997) Detection of p53 gene mutation by rapid PCR-SSCP and its association with poor survival in breast cancer. Int. J. Cancer 74: 642-647.

50. Norberg T, Klaar S, Karf G, Nordgren H, Holmberg L and Bergh J (2001) Increased p53 mutation frequency during tumor progression - results from a breast cancer cohort. Cancer Res. 61: 8317-8321.

51. Pharoah PD, Day NE and Caldas C (1999) Somatic mutations in the p53 gene and prognosis in breast cancer: a meta-analysis. Br. J. Cancer 80: 1968-1973.

52. Powell BL, Bydder S, Grieu F, Gnanasampanthan G, Elsaleh H, Seshadri R, Berns EM and lacopetta B (2001) Prognostic value of TP53 gene mutation in adjuvant treated breast cancer patients. Breast Cancer Res. Treat. 69: 65-68.

53. Miller LD, Smeds J, George J, Vega VB, Vergara L, Ploner A, Pawitan Y, Hall P, Klaar S, Liu ET and Bergh J (2005) From The Cover: an expression signature for p53 status in human breast cancer predicts mutation status, transcriptional effects, and patient survival. Proc. Natl. Acad. Sci. USA 102: 13550-13555.

54. Aas T, Borresen AL, Geisler S, Smith-Sorensen B, Johnsen H, Varhaug JE, Akslen LA and Lonning PE (1996) Specific P53 mutations are associated with de novo resistance to doxorubicin in breast cancer patients. Nat. Med. 2 : 811-814. 
55. Berns EM, van Staveren IL, Look MP, Smid M, Klijn JG and Foekens JA (1998) Mutations in residues of TP53 that directly contact DNA predict poor outcome in human primary breast cancer. Br. J. Cancer 77: 1130-1136.

56. Vogelstein B, Lane D and Levine AJ (2000) Surfing the p53 network. Nature 408: 307-310.

57. Kato S, Han SY, Liu W, Otsuka K, Shibata H, Kanamaru R and Ishioka C (2003) Understanding the function-structure and function-mutation relationships of p53 tumor suppressor protein by high-resolution missense mutation analysis. Proc. Natl. Acad. Sci. USA 100: 8424-8429.

58. Munro AJ, Lain S and Lane DP (2005) P53 abnormalities and outcomes in colorectal cancer: a systematic review. Br. J. Cancer 92: 434-444.

59. Geisler S, Lonning PE, Aas T, Johnsen H, Fluge O, Haugen DF, Lillehaug JR, Akslen LA and Borresen-Dale AL (2001) Influence of TP53 gene alterations and $\mathrm{c}$-erbB-2 expression on the response to treatment with doxorubicin in locally advanced breast cancer. Cancer Res. 61: 2505-2512.

60. Geisler S, Borresen-Dale AL, Johnsen H, Aas T, Geisler J, Akslen LA, Anker G and Lonning PE (2003) TP53 gene mutations predict the response to neoadjuvant treatment with 5-fluorouracil and mitomycin in locally advanced breast cancer. Clin. Cancer Res. 9: 5582-5588.

61. Elsaleh H, Powell B, McCaul K, Grieu F, Grant R, Joseph D and lacopetta B (2001) P53 alteration and microsatellite instability have predictive value for survival benefit from chemotherapy in stage III colorectal carcinoma. Clin. Cancer Res. 7: 1343-1349.

62. Bunz F, Hwang PM, Torrance C, Waldman T, Zhang Y, Dillehay L, Williams J, Lengauer C, Kinzler KW and Vogelstein B (1999) Disruption of p53 in human cancer cells alters the responses to therapeutic agents. J. Clin. Invest. 104: 263-269.

63. Buttitta F, Marchetti A, Gadducci A, Pellegrini S, Morganti M, Carnicelli V, Cosio S, Gagetti O, Genazzani AR and Bevilacqua G (1997) p53 alterations are predictive of chemoresistance and aggressiveness in ovarian carcinomas: a molecular and immunohistochemical study. Br. J. Cancer 75: 230-235.

64. Komarova EA, Krivokrysenko V, Wang K, Neznanov N, Chernov MV, Komarov PG, Brennan ML, Golovkina TV, Rokhlin OW, Kuprash DV, Nedospasov SA, Hazen SL, Feinstein E and Gudkov AV (2005) p53 is a suppressor of inflammatory response in mice. FASEB J. 19: 1030-1032.

65. Schrans-Stassen BH, Lue $H$, Sonnemans DG, Bernhagen $J$ and Post MJ (2005) Stimulation of vascular smooth muscle cell migration by macrophage migration inhibitory factor. Antioxid. Redox. Signal. 7: 1211-1216.

66. Fingerle-Rowson G, Petrenko O, Metz CN, Forsthuber TG, Mitchell R, Huss R, Moll U, Muller W and Bucala R (2003) The p53-dependent effects of macrophage migration inhibitory factor revealed by gene targeting. Proc. Natl. Acad. Sci. USA 100: 9354-9359.

67. Seril DN, Liao J, Yang GY and Yang CS (2003) Oxidative stress and ulcerative colitis-associated carcinogenesis: studies in humans and animal models. Carcinogenesis 24: 353-362.
68. Pikarsky E, Porat RM, Stein I, Abramovitch R, Amit S, Kasem S, GutkovichPyest E, Urieli-Shoval S, Galun E and Ben-Neriah Y (2004) NF-kappaB functions as a tumour promoter in inflammation-associated cancer. Nature 431: 461-466.

69. Sablina AA, Budanov AV, Ilyinskaya GV, Agapova LS, Kravchenko JE and Chumakov PM (2005) The antioxidant function of the p53 tumor suppressor. Nat. Med. 11: 1306-1313.

70. Aoki M, Nata T, Morishita R, Matsushita H, Nakagami H, Yamamoto $K$, Yamazaki K, Nakabayashi M, Ogihara T and Kaneda Y (2001) Endothelial apoptosis induced by oxidative stress through activation of NF-kappaB: antiapoptotic effect of antioxidant agents on endothelial cells. Hypertension 38: 48-55.

71. Davis BB, Dong $Y$ and Weiss RH (2003) Overexpression of p73 causes apoptosis in vascular smooth muscle cells. Am. J. Physiol. Cell Physiol. 284: C16-C23.

72. Lavezzi AM, Milei J, Grana DR, Flenda F, Basellini A and Matturri L (2003) Expression of c-fos, p53 and PCNA in the unstable atherosclerotic carotid plaque. Int. J. Cardiol. 92: 59-63.

73. Guevara NV, Kim HS, Antonova El and Chan L (1999) The absence of p53 accelerates atherosclerosis by increasing cell proliferation in vivo. Nat. Med. 5 : 335-339.

74. Merched AJ, Williams E and Chan L (2003) Macrophage-specific p53 expression plays a crucial role in atherosclerosis development and plaque remodeling. Arterioscler. Thromb. Vasc. Biol. 23: 1608-1614.

75. Duan W, Zhu X, Ladenheim B, Yu QS, Guo Z, Oyler J, Cutler RG, Cadet JL, Greig NH and Mattson MP (2002) p53 inhibitors preserve dopamine neurons and motor function in experimental parkinsonism. Ann. Neurol. 52 : 597-606.

76. Lavin MF (1999) ATM: the product of the gene mutated in ataxia-telangiectasia Int. J. Biochem. Cell Biol. 31: 735-740.

77. Gueven N, Becherel OJ, Kijas AW, Chen P, Howe O, Rudolph JH, Gatti R, Date $\mathrm{H}$, Onodera O, Taucher-Scholz G and Lavin MF (2004) Aprataxin, a novel protein that protects against genotoxic stress. Hum. Mol. Genet. 13: 10811093

78. Feng Z, Jin S, Zupnick A, Hoh J, de Stanchina E, Lowe S, Prives C and Levine AJ (2006) p53 tumor suppressor protein regulates the levels of huntingtin gene expression. Oncogene 25: 1-7.

79. Chattopadhyay B, Baksi K, Mukhopadhyay S and Bhattacharyya NP (2005) Modulation of age at onset of Huntington disease patients by variations in TP53 and human caspase activated DNase (hCAD) genes. Neurosci. Lett. 374: 8186.

80. Walter K, Warnecke G, Bowater R, Deppert W and Kim E (2005) tumor suppressor p53 binds with high affinity to CTG. CAG trinucleotide repeats and induces topological alterations in mismatched duplexes. J. Biol. Chem. 280: 42497-42507. 